\title{
Plasma-chemical synthesis of copper oxide nanoparticles in a low-pressure arc discharge
}

\author{
A.V. Uschakov ${ }^{a, b}$, I.V. $\operatorname{Karpov}^{a, b}$, A.A. Lepeshev ${ }^{a, b}{ }^{*}$, M.I. Petrov ${ }^{a, c}$ \\ ${ }^{a}$ Krasnoyarsk Scientific Center of the Siberian Branch of the Russian Academy of Science, \\ 660036, Krasnoyarsk, Russia \\ ${ }^{b}$ Siberian Federal University, 660041, Krasnoyarsk, Russia \\ ${ }^{c}$ Kirensky Institute of Physics, Russian Academy of Sciences, 660036, Krasnoyarsk, Russia \\ * Corresponding author. \\ E-mail address: sfu-unesco@mail.ru (A.A. Lepeshev)
}

Abstract. The influence of a pressure of gas mixture $\left(10\right.$ vol $\left.\% \mathrm{O}_{2}+90 \% \mathrm{~N}_{2}\right)$ on an average size of copper oxide nanoparticles, produced in the plasma of low pressure arc discharge, has been studied as a basic process variable. A correlation between the dependence of average particle size on gas mixture pressure and the dependence of discharge gap voltage on product of interelectrode distance by a gas mixture pressure, has been found. The estimation was carried out by means of X-ray diffraction (XRD) and high-resolution transmission electron microscopy (HRTEM). A mathematical model of the cathode region, which shows the applicability of the similarity theory to the low pressure arc discharge, has been represented.

Keywords: copper oxide nanoparticles, low pressure arc discharge, plasma-chemical synthesis, Paschen curve, similarity parameter.

\section{Introduction}

Copper oxide with nanosized particles and films are useful materials in a variety of applications such as photovoltaic devices [1,2], electrochromic devices [3], thin film transistors [4], and chemical sensors[5], owing to their moderate band gap, low cost production, and high optical transparency. Copper oxide nanoparticles are commonly synthesized by wet chemical 
processes [6-8]. However, it is not easy to ensure the homogeneity and crystallinity of the nanoparticles when fabricated through such methods. This is because wet chemical processes involve low temperatures. In contrast to wet chemical processes, thermal plasma-based ones involve the evaporation of the constituent metals at temperatures higher than $10,000 \mathrm{~K}$, followed by the rapid condensation of the gas phases [9-13]. Thermal plasma-based processes have other advantages over wet chemical processes. One is that, using these processes, it is possible to prevent the end products from being contaminated by impurities; this is not the case with wet chemical processes $[14,15]$. So far, a number of thermal plasma based processes have been developed. Among them, the vacuum arc plasma evaporation (VAPE) method is commonly used for the deposition of thin films [16] and the fabrication of nanoparticles [17-22]. We recently reported that particle phases with different stoichiometries, including $\mathrm{Cu} 2 \mathrm{O}$ and $\mathrm{CuO}$, can be produced by varying the deposition conditions, such as oxygen partial pressure, discharge power, processing pressure, and substrate temperature [23].

According to the previous research, the properties of powders produced in the plasma arc discharge of low pressure, depend mainly on the gas mixture pressure in a plasma-chemical reactor [24]. Dispersion of produced powders depends mainly on two processes: evaporation and ionization of a liquid metal on a cathode surface, and condensation form a plasma-vapor phase. In a sufficiently high vacuum of $\sim 10^{-1}$ Pa powder consists of particles of two types: spherical particles with size of $0.5-3 \mu \mathrm{m}$, wherein fine condensate dropped, and spherical particles with size of $0.5-10 \mu \mathrm{m}$, which represent agglomerates of smaller particles with size about $0.1 \mu \mathrm{m}$ [24,25]. The percentage of nanopowders in this case is negligible. The experimental results clearly show the advantages of powders obtained at different pressures of the gas mixture. Change of the pressure in the plasma-chemical reactor does not lead to change of the particle size distribution function. This shows predominantly thermal nature of arc powders synthesis, where the particles are formed due to vapor condensation. The purpose of this work is to define the mechanism influencing the pressure of gas mixture in a plasma-chemical reactor on an 
average size and morphology of copper oxide nanoparticles, produced in plasma arc discharge of low pressure.

\section{Materials and Methods}

The synthesis of $\mathrm{CuO}$ nanopowder was carried out in the plasma-chemical reactor described in [26]. The arc evaporator, used in the process, had the following characteristics: a current arc of $100 \mathrm{~A}$, an intensity of the longitudinal magnetic field excited by the focusing coil on the surface of the cathode, of $80 \mathrm{~A} / \mathrm{m}$, a distance between the cathode and the anode of $d=50 \mathrm{~mm}$. A rod, made from copper (99.99\%) with a diameter of $80 \mathrm{~mm}$ and a length of $100 \mathrm{~mm}$, was used as a cathode. It was placed on a water cooled copper current lead. In order to begin plasma chemical reactions, the gas of the chamber was preliminary pumped out to a pressure of $\mathrm{p}=1 \mathrm{mPa}$ and then the chamber was filled by gas mixture containing 10 vol. $\% \mathrm{O}_{2}$ of the amount of supplied plasma-generating gas $\mathrm{N}_{2}$. In order to study the pressure influence, nanoparticles were synthesized at basic pressures of 10, 50, 80, 140 and $200 \mathrm{~Pa}$. Gas control was carried out continuously by means of a gas flow regulator and a vacuum manometer. Oxygen was supplied to the reactor in order to form a uniform layer around the plasma torch. The reaction products were collected during $10 \mathrm{~min}$ on a hemispherical collector made of water-cooled stainless steel, placed at a distance of $0.12 \mathrm{~m}$ from the cathode.

Morphological composition of the samples has been studied by transmission electron microscope JEOL JEM-2100. The analysis of dispersion distribution was performed by the method described in [27]. The study of the phase composition of the samples has been carried out using X-ray diffractometer Advance D8 in CuKa monochromatic radiation. Scanning was performed at room temperature in the range of angles at 20-120 deg with $2 \theta$ step of $0.06 \mathrm{deg}$. The microstructural characteristics and the elementary cell parameters have been determined using X-ray full-profile analysis by the Rietveld method. 


\section{Results and discussion}

Figure 1 shows the high-resolution transmission electron microscopy images, the diagram of particle distribution and the size distribution function of the copper oxide nanoparticles produced under the pressure of the gas mixture $\left(10\right.$ vol. $\left.\% \mathrm{O}_{2}+90 \% \mathrm{~N}_{2}\right)$ of $80 \mathrm{~Pa}$.

It is easy to conclude from the above results, that the produced powder represents strongly agglomerated particles of spherical shape. The particle size ranges from 5 to $20 \mathrm{~nm}$. The combination of normal and lognormal size distribution are characterized for these particles. This suggests two competing mechanisms of particle condensation from steam-plasma phase: cluster condensation and steam condensation. The mixed distribution function represents the sum of functions of lognormal and normal distribution with adjustable parameters. It can be seen from the figure above, that this function describes the particle size distribution much better. The average particle size was $9.4 \mathrm{~nm}$, the mean mass particle size was $10.2 \mathrm{~nm}$ and the standard deviation was 1.18 .

Nanopowders are characterized by high surface energy, which is balanced out by a significant aggregation of powders, which causes a significant decrease in the specific surface. It is well known [27] that particle size distribution function directly corresponds to the process of nanoparticles formation. The size distribution function can differ for various methods of nanopowders production significantly. There are two main mechanisms for nanoparticles formation: diffusive and coagulative.

While the nanoparticle chemical processes always accompany corresponding synthesis in plasmachemical reactor, the size distribution function of nanoparticles becomes more complicated because of the products of these reactions. The morphology of the particles also becomes more complicated and particles of different chemical composition are formed. Besides, reciprocal diffusion of nanoparticles in the condensed phase takes place. Under sufficiently high temperature, multiple processes can occur simultaneously, forming the strong coupling between nanoparticles. The experimental data are described by a normal distribution while prevailing the 
layered growth of nanoparticles due to the adsorption of atoms and diffusion processes during the atomic mass transfer at the interface [28].

Figure 2 shows the X-ray diffraction patterns of nanoparticles synthesized at the pressures of gas mixture of $\left(10\right.$ vol. $\left.\% \mathrm{O}_{2}+90 \% \mathrm{~N}_{2}\right)$ for $2 \theta$ values in the range from 25 to $65 \mathrm{deg}$.

The diffraction patterns of nanoparticles clearly show reflexes (JCPDS data, No. 05-0667), corresponding to cuprite structure of $\mathrm{Cu}_{2} \mathrm{O}$, space group $\mathrm{Pn} 3 \mathrm{~m}$. Other crystalline structures have not been found.

Figure 3 shows the results of X-ray studies using PowderCell 2.4 software. The dependence of the average size of coherent scattering areas on the pressure of the various gas mixtures is shown.

The dependence of the average size of coherent scattering region (CSR) on gas mixture pressure is shown below. The influence of the pressure on the average size of powders was studied over a wider range of values, but this image shows only the most representative results. For example, under the pressure of the gas mixture of $1 \mathrm{~Pa}$ or less, the produced powders are widely ranged in size from $50 \mathrm{~nm}$ to $10 \mu \mathrm{m}$. In order to produce powders under the pressures above $200 \mathrm{~Pa}$, an increase in voltage is necessary to maintain stable arcing. It causes the transition of the diffuse plasma column to the typical arch-shaped column and appearance of anchoring cathode spots and their grouping, which lead to a cathode splashing with large drops.

The microscopic studies showed that $\mathrm{Cu}_{2} \mathrm{O}$ nanoparticles, produced at a pressure of $10 \mathrm{~Pa}$, have a large size distribution (from 10 to $150 \mathrm{~nm}$ ), are highly agglomerated and cannot be disaggregated. Regarding these facts, the particle size analysis is significantly more difficult. Comparison of Figures 1 and 3 shows a good agreement of XRD and TEM studies of $\mathrm{Cu}_{2} \mathrm{O}$ nanoparticles produced under the pressure of $80 \mathrm{~Pa}$.

Figure 3 shows the dependence of discharge gap voltage $U$ on the gas mixture pressure $p$ and the distance $\mathrm{d}$ between the cathode and the anode in the chamber of plasma chemical reactor. 
It is obvious from the figure, that the behavior of all the curves is the same and represents a gradual decrease of voltage to a bending point and further increase up to the maximum value of the gas mixture pressure. The comparison of curves of the average particle sizes and the discharge gap voltage allows concluding that the optimum value of the pressure of the gas mixture for producing $\mathrm{Cu}_{2} \mathrm{O}$ nanoparticles with minimum average sizes is in the range of 70-100 Pa. The graphs also show that changing the pressure in the plasma-chemical reactor allows controlling the average size of nanoparticles.

The behavior of the curves, showing the function of voltage on the discharge gap, is similar in shape to the curves, showing the mean values of the size of powders. It should be mentioned that the measurement of the voltage on the discharge gap, in the pressure range of gas mixture from 10-3 $\mathrm{Pa}$ to $50 \mathrm{~Pa}$, appears to be of negligible influence of the pressure on the plasma processes and processes of the current transfer. However, under the pressure of gas mixture over $50 \mathrm{~Pa}$ up to $200 \mathrm{~Pa}$ the behavior of the curves $U(p d)$ almost completely coincides with the theory of similarity.

The approximating curve is defined by the known equation [29]

$$
U(p d)=\frac{B p d}{\ln [A p d / \ln (1+1 / \gamma)]}
$$

where $\mathrm{A}$ and $\mathrm{B}$ are constants depending on the type of gas. They are found in such a way to achieve the best correlation with the experimental results, $\gamma$-the second Townsend coefficient depending on the cathode material, and generally on $p d$. However, $\gamma$ is included twice under the logarithm sign and insignificantly influences on the function $U(p d)$ in the range being critical here. The function $U(p d)$ shows its minimum in the point of $(p d)_{\min }=\frac{\exp (1)}{A} \ln \left(1+\frac{1}{\gamma}\right)$.

The expression (1) can be shown in a simple form: 


$$
U(p d)=\frac{B p d}{\ln (p d)+C}
$$

where $C=\ln A-\ln (\ln (1+1 / \gamma))$.

The deviation of the left branch of the curve $U(p d)$ from classical Paschen curve is due to a large number of factors. In this case, all the measurements were performed by means of a pointer gage, which averages the voltage fluctuations on the discharge gap, which varies widely during arcing. Measurements using a high-speed oscillograph with a resolution of at least higher than 10-9 s showed that the amplitude of noise oscillations of voltage varies from 1 to $150 \mathrm{~V}$ under the gas mixture pressure less than $10 \mathrm{~Pa}[30,31]$. The average pulse duration was equal to $30 \mathrm{~ns}$. Such behavior of the voltage through the discharge gap is correlated with a large range of sizes of the nanoparticles produced at this pressure.

In this respect, the peak value of the pulse voltage corresponds the left branch of the Paschen curve, and it means that among the process parameters, determining the synthesis of nanopowders, only one parameter should be investigated: it is the pressure of the gas mixture in the plasma chemical reactor. Besides, it can be assumed that a continuous arc discharge consists of a series of spark bursts of discharge gap in the cathode zone having a characteristic size of $10^{-}$ ${ }^{4} \mathrm{~m}$ filled with a metal high-pressure steam, it has a duration of 1-30 ns. Therefore, in order to simulate physical processes in the cathode region, the well-known methods of sparks avalanchestreamer mechanisms can be used [32].

The difference from a gas burst is that the main voltage decrease is concentrated mainly in the cathode spot and the burst is carried out in the pre-ionized metal vapor. The high rate of scattering of metal vapor jets $\left(10^{4} \mathrm{~m} \cdot \mathrm{s}^{-1}\right)$ significantly spoils the burst condition that is the main cause of high amplitude of impulse constituent of the discharge voltage. Gas in the plasmachemical reactor acts as a buffer, it partially prevents the output of the metal vapor, ions and of electrons from the cathode spot zone. Therefore, the voltage fluctuations at a pressure higher than $50 \mathrm{~Pa}$ decrease to $1 \mathrm{~V}$ sharply. This fact is confirmed by the experiments described in 
$[33,34]$, that show that the increase in a pressure leads to a significant reduction in a velocity of electrons and vapor-plasma jets.

While all the processes in the cathode region have a characteristic duration of 1-100 ns, so the solving the ionization problem requires only the solving the drift-diffusion Nernst-PlanckEinstein equations, supplemented by the Poisson equation, neglecting the processes of plasma formation, electrical neutrality, etc.

$$
\left\{\begin{array}{c}
\frac{\partial n_{e}}{\partial t}+\operatorname{div}\left[-D_{e} \nabla n_{e}-b_{e} n_{e} \vec{E}\right]=v_{i o n}(E) n_{e}-v_{a t t}(E) n_{e}-c_{e e} n_{e} n_{+}+S_{p h}+S_{u n} \\
\frac{\partial n_{+}}{\partial t}+\operatorname{div}\left[-D_{+} \nabla n_{+}+b_{+} n_{+} \vec{E}\right]=v_{i o n}(E) n_{e}-c_{e e} n_{+} n_{e}+S_{p h}+S_{u n} \\
\Delta \varphi=-\left(\frac{e}{\varepsilon_{0}}\right)\left(n_{+}-n_{e}\right) ; \vec{E}=-\nabla \varphi
\end{array}\right.
$$

where $n_{e}, n_{+}$are concentrations of electrons and ions, respectively, $\varphi$ is an electric potential.

The function of source for the electrons takes into account appearance of electrons caused by impact ionization with the frequency of $v_{i o n}(E)$, as well as losses of electrons during the adherence with a frequency of $v_{\text {att }}(E)$, and losses during the electrons and ions recombination with the coefficient of $c_{e e}$. The function of source for positive ions takes into account their appearance caused by the impact ionization and their losses while recombination of the electrons and ions (factor $c_{e e}$ is set for the previous value).

The system of differential equations has been solved in the Comsol software package by the finite element method. In this model the nonstationary one-dimensional problem with a uniform field was solved. The electrons were emitted from the cathode and this process was constant in time. Ionization and recombination processes took place in the gap and were taken into account as a reaction rate. The initial concentration of the ions was defined according to the potential decrease across the gap. The initial concentration of ions and electrons in the discharge gap was zero. 
The figures show the development of the arc discharge at different points in time. Fig. 4, a) shows the change in the concentration of electrons emitted by the cathode in the process of moving to the ion cloud.

The number of electrons in the cathode region gradually increases under the influence of impact ionization, and the radius of the cloud increases due to the diffusion. The bulk charge is too small (Fig. 5, a) for significant changing the electric field (Fig. 5, b) that is the initial phase. Two electron density peaks are clearly seen during the development of the arc discharge. The first peak is the main and is observed in the cathode region, while the second is observed near the ion cloud. The appearance of the second peak is caused by the first few electrons reached the ion cloud. Because of the diffusion some separate electrons reach it before all the other electrons. These electrons lead to a rapid increase in the concentration right at the ion cloud due to a high field intensity near ions and as a consequence, due to a high ionization rate. This peak rapidly exceeds the first one by magnitude and becomes noticeable on the background in the moment of time $t=4.7 \mathrm{~ns}$. Nevertheless, since the rate of ionization processes is much higher than electron drift rate, the first peak begins to grow rapidly and they become equal at a moment of time of 4.8 ns.

Due to intense ionization in the cathode layer, the uncompensated positive charge is gradually accumulated (Fig. 5, a), and begins to influence the external electric field (Fig. 5, b), displacing it from the zone of increased concentration of electrons to the boundary of this zone. The zone with increased ionization is also moved there (Fig. 5, c). This leads to increasing the concentrations of ionized particles before the boundaries (Fig. 4, b). Thus the zone of high concentration of charged particles changes its borderies again. Electron cloud starts to spread towards the cathode from the ion cloud. From this point it can be called "reflected" electron cloud. At this time the state of the zone with high concentration of electrons changed to the plasma state -the field is displaced from here, the bulk charge is practically zero. 
The above-stated correlates the current understanding of the formed cathode spot structure, which is characterized by the plasma channel, and its field is displaced to the outer boundary of the channel [35]. The field peak in this case is located at the front boundary between the cathode zone and the ion cloud. If the arc is developed in the field formed by the electrodes, practically not having changed it, then since the formation of the plasma channel the conductive zone is developed at the expense of the newly formed plasma, deforming this field and forming a "plasma reaction zone". These peaks become new centers of the ionization processes where emission electrons are involved as seed electrons. A new plasma zone with high concentration of electrons ionization is generated as a result of ionization, which lengthens and narrows the plasma channel, displacing the electric field. The ionization wave moves as described above.

The rate of arc current increase is the rate of ionization wave, which can be much higher than a rate of ions move [32]. The field intensity in the plasma channel is less than in front of it. The field intensity it is too low and the impact of ionization in the channel does not occur. The key role is played by the loss of electrons due to recombination process.

In this respect, the arc discharge represents a series of spark discharges along with the formation of an electron avalanche and streamer channel. Therefore, the similarity theory with a parameter $p d$ is applicable to the low-pressure arc discharge. In this way, in order to develop a technology only the pressure of the gas mixture should be taken into account. A spark discharge in cathode spot differs from a spark discharge in gases is that the process is developed in a narrow cathode region in the high-pressure metal vapor. However, the ionization processes are also developed mainly due to the impact ionization.

\section{Conclusion}

The particularities of plasma chemical low pressure synthesis of copper oxide nanoparticles related to the mixed nature of condensation have been revealed. The correlation between the dependence of the average particle size found by means of X-ray, on the gas mixture pressure, 
and the dependence of the discharge gap voltage of arc plasma generator on the parameter $p d$, has been established. This fact shows a common mechanism of nanoparticles synthesis in a low pressure arc discharge for the cooled cathode. The optimum pressure for the synthesis of nanomaterials in plasma-arc evaporator is in the range of 70-100 $\mathrm{Pa}$. The represented mathematical model allows using the avalanche-streamer mechanism to explain the ionization processes in a cathode region of low pressure arc discharge.

\section{Acknowledgments}

The work was performed with a support of the grant of the Russian Science Foundation (Project No. 16-19-10054) 


\section{References}

[1] F.K. Mugwang'a, P.K. Karimi, W.K. Njoroge, O. Omayio, S.M. Waita, Optical characterization of Copper Oxide thin films prepared by reactive $d c$ magnetron sputtering for solar cell applications, Int. J. Thin Film Sci. Tec. 2 (2013) 15-24.

[2] N. Gupta, R. Singh, F. Wu, J. Narayan, C. McMillen, G.F. Alapatt, K.F. Poole, S.J. Hwu, D. Sulejmanovic, M. Young, G. Teeter, H.S. Ullal, Deposition and characterization of nanostructured $\mathrm{Cu}_{2} \mathrm{O}$ thin-film for potential photovoltaic applications, J. Mater. Res. 28 (2013) 1740-1746. doi: http://dx.doi.org/10.1557/jmr.2013.150.

[3] T.J. Richardson, J.L. Slack, M.D. Rubin, Electrochromism in copper oxide thin films, Electrochimica Acta 46 (2001) 2281-2284. doi: 10.1016/S0013-4686(01)00397-8.

[4] J.W. Park, K.J. Baeg, J. Ghim, S.J. Hang, J.H. Park, D.Y. Kim, Effects of Copper Oxide/Gold Electrode as the Source-Drain Electrodes in Organic Thin-Film Transistors Semiconductor Devices, Materials, and Processing, Electrochem. Solid-State Lett. 10 (2007) H340-H343. doi: 10.1149/1.2774683.

[5] D. Zappa, E. Comini, R. Zamani, J. Arbiol, J.R. Morante, G. Sberveglieri, Preparation of copper oxide nanowire-based conductometric chemical sensors, Sensors and Actuators B: Chemical 182 (2013) 7-15. doi: 10.1016/j.snb.2013.02.076.

[6] L. Liu, K. Hong, T. Hu, M. Xu, Synthesis of Aligned Copper Oxide Nanorod Arrays by a Seed Mediated Hydrothermal Method, J of Alloys and Compounds 511 (2012) 195-197. doi: 10.1016/j.jallcom.2011.09.028.

[7] S. Srivastava, M. Kumar, A. Agrawal, S.K. Dwivedi, Synthesis and Characterisation of Copper Oxide nanoparticles, IOSR Journal of Applied Physics (IOSR-JAP) 5 (2013) 61-65.

[8] Shah M. Asharf, M.S. Al-Ghamdi, Preparation of Copper $(\mathrm{Cu})$ and Copper Oxide $\left(\mathrm{Cu}_{2} \mathrm{O}\right)$ Nanoparticles under Supercritical Conditions, Materials Sciences and Application 2 (2011) 977980. doi: $10.4236 / \mathrm{msa} .2011 .28131$. 
[9] R.K. Swarnkar, S.C. Singh, R. Gopal, Effect of aging on copper nanoparticles synthesized by pulsed laser ablation in water: structural and optical characterizations, Bull. Mater. Sci. 34 (2011) 1363-1369. doi: 10.1007/s12034-011-0329-4.

[10] J. Musil, P. Baroch, High-rate pulsed reactive magnetron sputtering of oxide nanocomposite coatings, Vacuum 87 (2013) 96-102. doi: 10.1016/j.vacuum.2012.02.023.

[11] K. Nadeem, H. Krenn, T. Traußnig, R. Würschum, D.V. Szabó, I. Letofsky-Papst, Spinglass freezing of maghemite nanoparticles prepared by microwave plasma synthesis, J. Appl. Phys. 111 (2012) 113911. doi: http://dx.doi.org/10.1063/1.4724348.

[12] P. Synek, O. Jašek, L. Zajíčková, Study of Microwave Torch Plasmachemical Synthesis of Iron Oxide Nanoparticles Focused on the Analysis of Phase Composition, Plasma Chem. Plasma Process. 34 (2014) 327. doi: 10.1007/s11090-014-9520-x.

[13] P. Lei, A. Boies, S. Calder, S. Girshick, Thermal Plasma Synthesis of Superparamagnetic Iron Oxide Nanoparticles, Plasma Chem. Plasma Process. 32 (2012) 519. doi: 10.1007/s11090-012-9364-1.

[14] Y. Xua, J. Yang, M. Demura, T. Hara, T. Hirano, Y. Matsushita, Tanaka, Y. Katsuya, Fabrication of $\mathrm{Ni}-\mathrm{Al}$ nanoparticles via vacuum arc plasma evaporation and their catalytic properties for CO oxidation, Applied Catalysis A: General 478 (2014) 165-174. doi: 10.1016/j.apcata.2014.03.027.

[15] M. Zhang, X. Tu, J. Wang, T. Fang, Y. Wang, X. Xu, M. Zhang, Y. Chen, Hydrothermal syntheses of $\mathrm{CuO}, \mathrm{CuO} / \mathrm{Cu}_{2} \mathrm{O}, \mathrm{Cu}_{2} \mathrm{O}, \mathrm{Cu}_{2} \mathrm{O} / \mathrm{Cu}$ and $\mathrm{Cu}$ microcrystals using ionic liquids, Chemical Research in Chinese Universities 32 (2016) 530-533. doi: 10.1007/s40242016-6037-2.

[16] V.Yu. Novikov, I.Yu. Goncharov, V.S. Zakhvalinskii, A.Y. Kolpakov, M.B. Ivanov, D.A. Kolesnikov, The electrical properties of coating obtained by vacuum arc deposition, Results in Physics 5 (2015) 72-73. 
[17] A.A. Ashkarran, M. Kavianipour, S.M. Aghigh, S.A. Ahmadi Afshar, S. Saviz, A. Iraji Zad, On the Formation of $\mathrm{TiO}_{2}$ Nanoparticles Via Submerged Arc Discharge Technique: Synthesis, Characterization and Photocatalytic Properties, J Clust Sci. 21 (2010) 753. doi: 10.1007/s10876-010-0333-7.

[18] A.V. Ushakov, I.V. Karpov, A.A. Lepeshev, L.Yu. Fedorov, A.A. Shaikhadinov, Plasmachemical Synthesis and Basic Properties of $\mathrm{CoFe}_{2} \mathrm{O}_{4}$ Magnetic Nanoparticles, Technical Physics 61 (2016) 103. doi: 10.1134/S1063784216010230.

[19] A.V. Ushakov, I.V. Karpov, A.A. Lepeshev, M.I. Petrov, Enhancing of magnetic flux pinning in $\mathrm{YBa}_{2} \mathrm{Cu}_{3} \mathrm{O}_{7-\mathrm{x}} / \mathrm{CuO}$ granular composites, J. Appl. Phys. 118 (2015) 023907. doi: http://dx.doi.org/10.1063/1.4926549.

[20] A.V. Ushakov, I.V. Karpov, A.A. Lepeshev, M.I. Petrov, L.Yu. Fedorov, Specific Features of the Behavior of Electroarc $\mathrm{CuO}$ Nanoparticles in a Magnetic Field. Physics of the Solid State 57 (2015) 919-923. doi: 10.1134/S1063783415050303.

[21] A.V. Ushakov, I.V. Karpov, A.A. Lepeshev, M.I. Petrov, L.Yu. Fedorov, Study of Magnetic Flux Pinning in Granular $\mathrm{YBa}_{2} \mathrm{Cu}_{3} \mathrm{O}_{7-\mathrm{y}} /$ nanoZrO 2 Composites, JETP Letters 99 (2014) 99-103. doi: 10.1134/S002136401402009X.

[22] A.A. Lepeshev, I.V. Karpov, A.V. Ushakov, L.Yu. Fedorov, A.A. Shaikhadinov, Synthesis of Nanosized Titanium Oxide and Nitride Through Vacuum Arc Plasma Expansion Technique, International Journal of Nanoscience $15 \quad$ (2016) 1550027 doi: http://dx.doi.org/10.1142/S0219581X15500271.

[23] A.V. Ushakov, I.V. Karpov, A.A. Lepeshev, S.M. Zharkov, The influence of oxygen concentration on the formation of $\mathrm{CuO}$ and $\mathrm{Cu}_{2} \mathrm{O}$ crystalline phases during the synthesis in the plasma of low pressure arc discharge, Vacuum 128 (2016) 123-127. doi: 10.1016/j.vacuum.2016.03.025. 
[24] L.Yu. Fedorov, I.V. Karpov, A.V. Ushakov, A.A. Lepeshev, Influence of Pressure and Hydrocarbons on Carbide Formation in the Plasma Synthesis of TiC Nanoparticles, Inorganic Materials 51 (2015) 25-28. doi:10.1134/S0020168515010057.

[25] A.V. Ushakov, I.V. Karpov, A.A. Lepeshev, Influence of the Oxygen Concentration on the Formation of Crystalline Phases of $\mathrm{TiO}_{2}$ during the Low-Pressure Arc-Discharge Plasma Synthesis, Technical Physics 86 (2016) 260-264. doi: 10.1134/S1063784216020262.

[26] A.V. Ushakov, I.V. Karpov, A.A. Lepeshev, Influence of the Oxygen Concentration on the Formation of Crystalline Phases of $\mathrm{ZrO}_{2}$ Nanoparticles during the Low-Pressure ArcDischarge Plasma Synthesis, Physics of the Solid State 57 (2015) 2320-2322. doi: $10.1134 / \mathrm{S} 1063783415110359$.

[27] I.V. Karpov, A.V. Ushakov, L.Yu. Fedorov, A.A. Lepeshev, Method for Producing Nanomaterials in the Plasma of a Low Pressure Pulsed Arc Discharge, Technical Physics 84 (2014) 559-563. doi: 10.1134/S1063784214040148.

[28] Klark E. R., Eberkhardt K. N. Microscopic methods of materials investigation: Moscow, 2007. - 376 p.

[29] D.I. Ryzhonkov, Ultra-dispersed medium. Production of nanopowders by chemical dispersion and their properties. Moscow: Education, 2007. P. 32-34.

[30] F. Paschen, Ueber die zum Funkenübergang in Luft, Wasserstoff und Kohlensäure bei verschiedenen Drucken erforderliche Potentialdifferenz, Annalen der Physik und Chemie 5 (1889) 273.

[31] D.I. Proskurovsky, V.F. Puchkarev, The formation of new emission centers on the cathode during electric current commutation in vacuum. 1. Patterns of formation of new emission centers, Technical Physics 49, (1979) 2611-2618.

[32] A.V. Ushakov, I.V. Karpov, A.A. Lepeshev, Modeling of metal vapor ionization processes in the cathode spot of a vacuum arc, Vestnik SibGAU. 16 (2015) 983-989.

[33] E.D. Losansky, O.B. Firsov, Theory of sparks. Moscow: Atomizdat. 1975. 
[34] A.S. Bugaev, V.I. Gushenets, A.G. Nikolaev, E.M. Oks, G.Yu. Yushkov, Study of directed ion velocities in a vacuum arc by an emission method, Technical Physics 45 (2000) 1135. doi: 10.1134/1.1318098.

[35] W.D. Davis, H.C. Miller, Analysis of the Electrode Products Emitted by dc Arcs in a Vacuum Ambient, J. Appl. Phys. 40 (1969) 2212. doi: http://dx.doi.org/10.1063/1.1657960.

[36] E. Hantzsche, On the inevitability of non-stationary vacuum arc spot models Phys. Lett. A 50 (1974) 219-220. doi:10.1016/0375-9601(74)90797-X. 


\section{Figure captions page}

FIG. 1. TEM images, histograms and size distribution function of copper oxide nanopowders produced under the pressure of the gas mixture $\left(10\right.$ vol. $\left.\% \mathrm{O}_{2}+90 \% \mathrm{~N}_{2}\right)$ of $80 \mathrm{~Pa}$

FIG. 2. The XRD patterns of copper oxide nanopowders produced at different pressures of the gas mixture (10 vol.\% $\left.\mathrm{O}_{2}+90 \% \mathrm{~N}_{2}\right)$

FIG. 3. Dependences of the average particle size of $\mathrm{Cu}_{2} \mathrm{O}$, calculated based on $\mathrm{X}$-ray, on gas mixture pressure of $\left(10 \mathrm{vol} . \% \mathrm{O}_{2}+90 \% \mathrm{~N}_{2}\right)$ and the discharge gap voltage with copper cathode on $p d$ parameter

FIG. 4. Concentration of electrons $a$ ) and ions and $b$ ) in the cathode region at different moments of time

FIG. 5. Distribution of the space charge density $a$ ), electric field strength $b$ ) and ionization intensity $c$ ) in the cathode region at different moments of time 

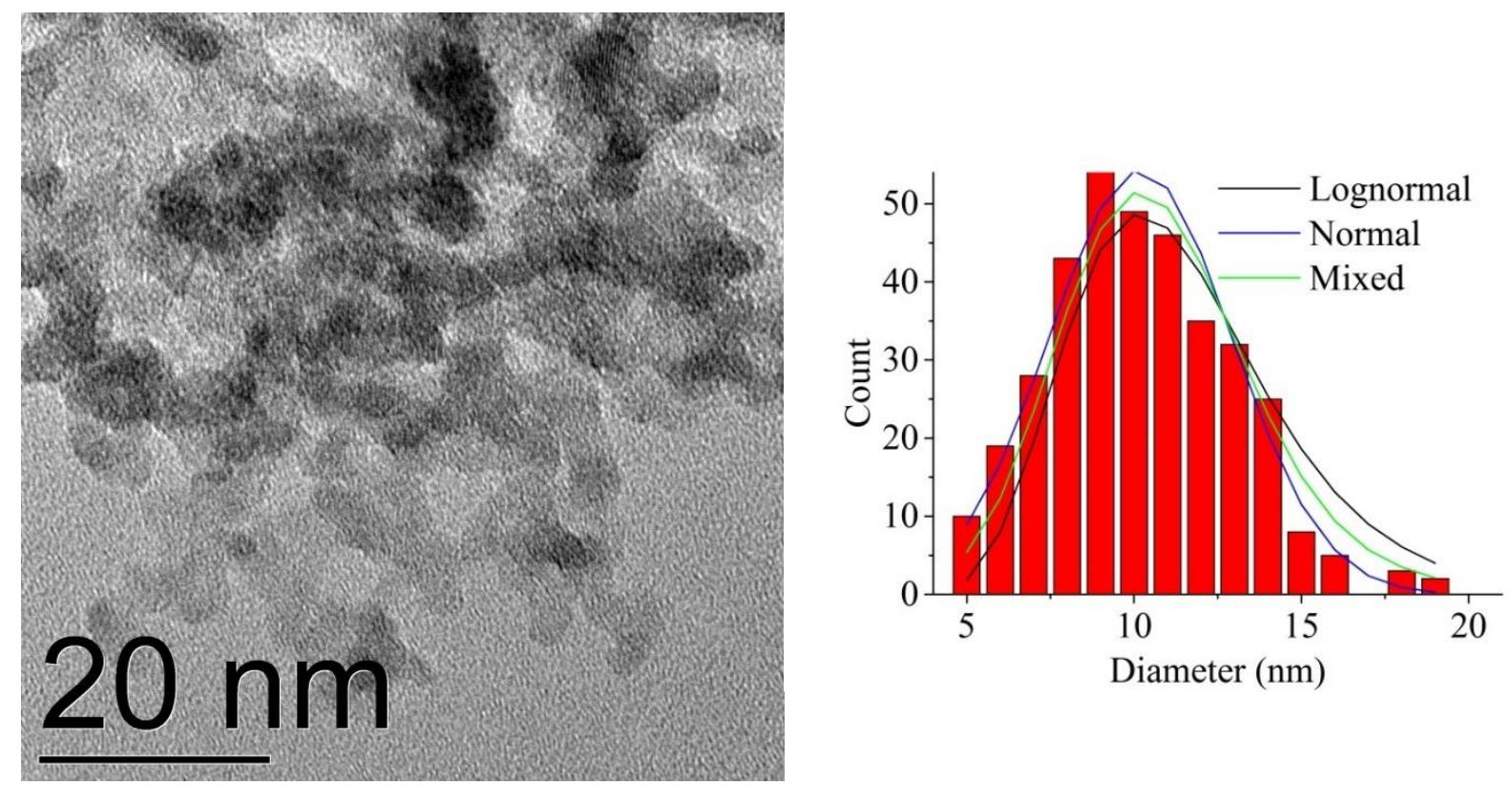

FIGURE 1. 


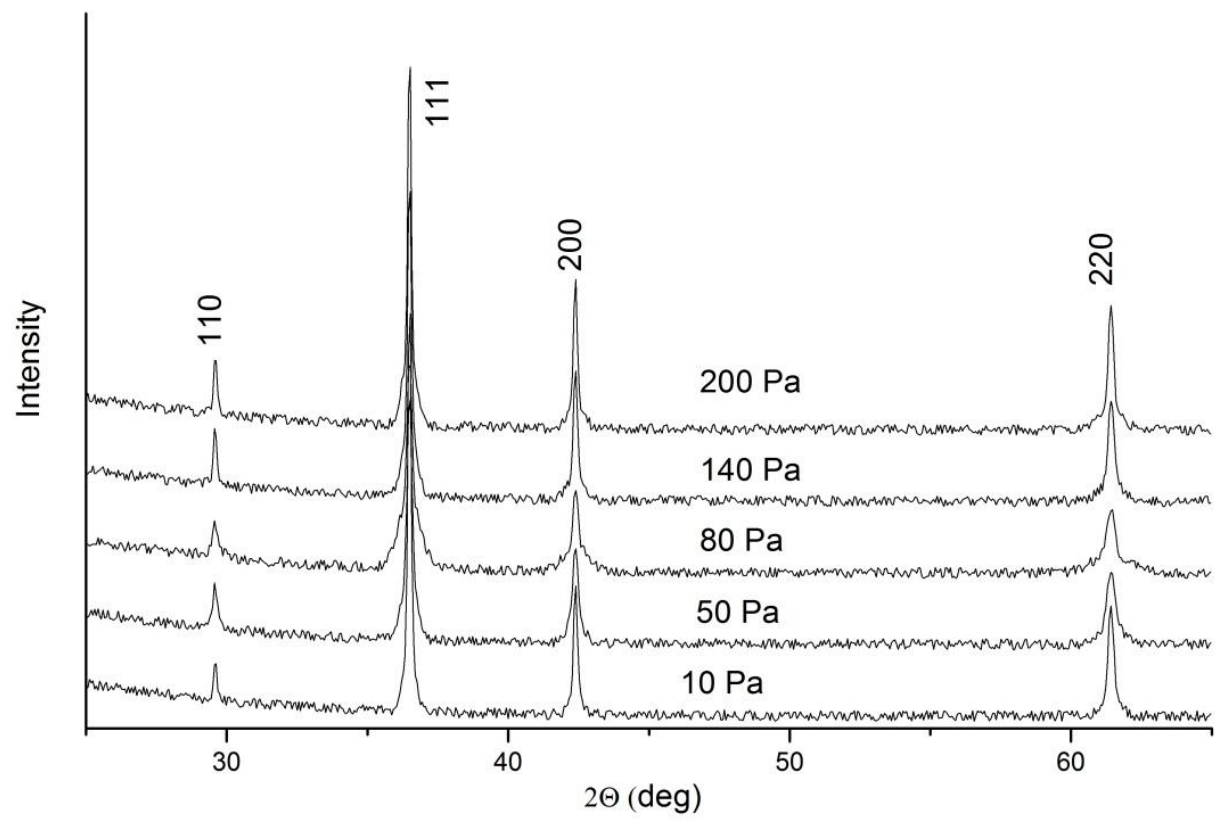

FIGURE 2. 


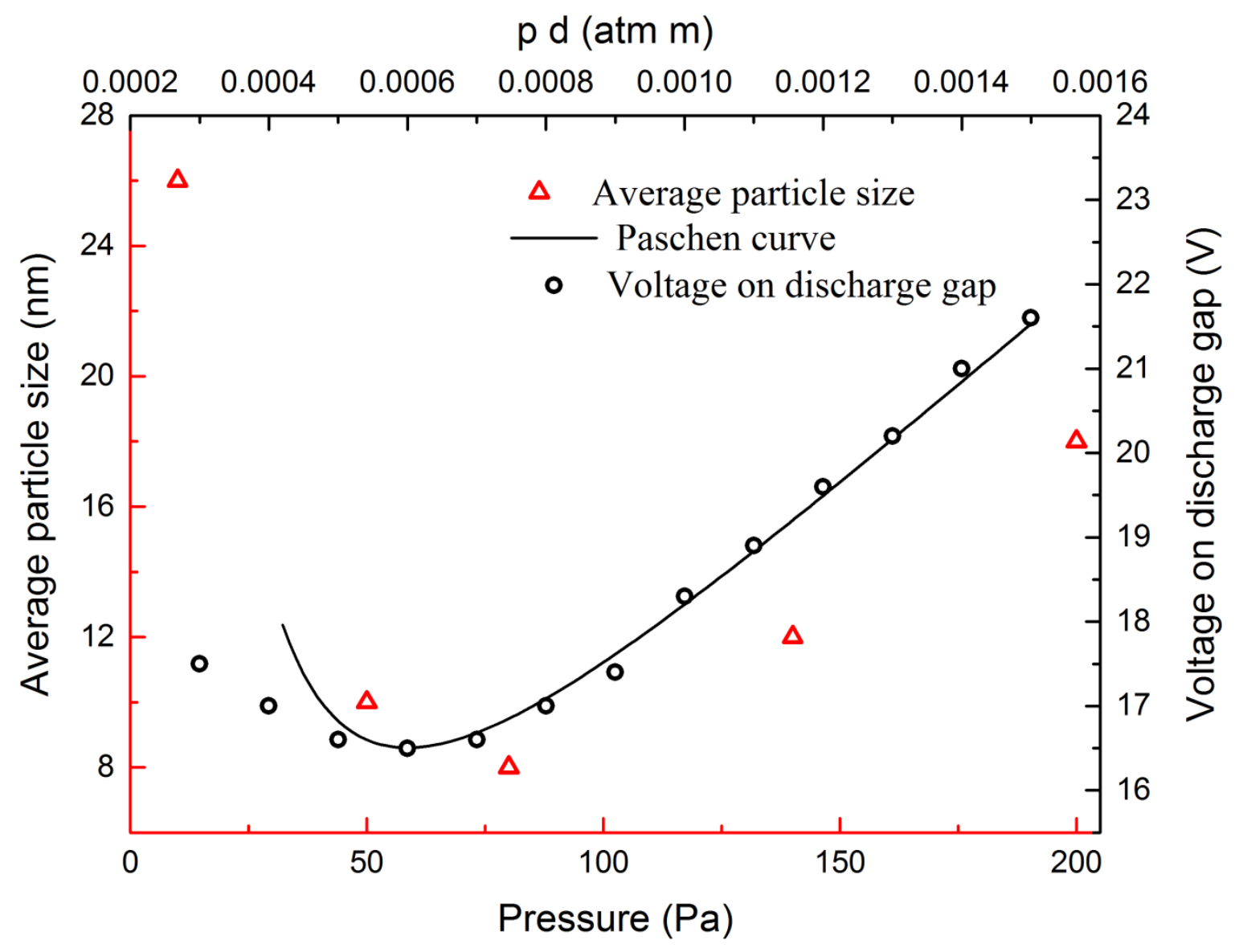

FIGURE 3. 
$\square$

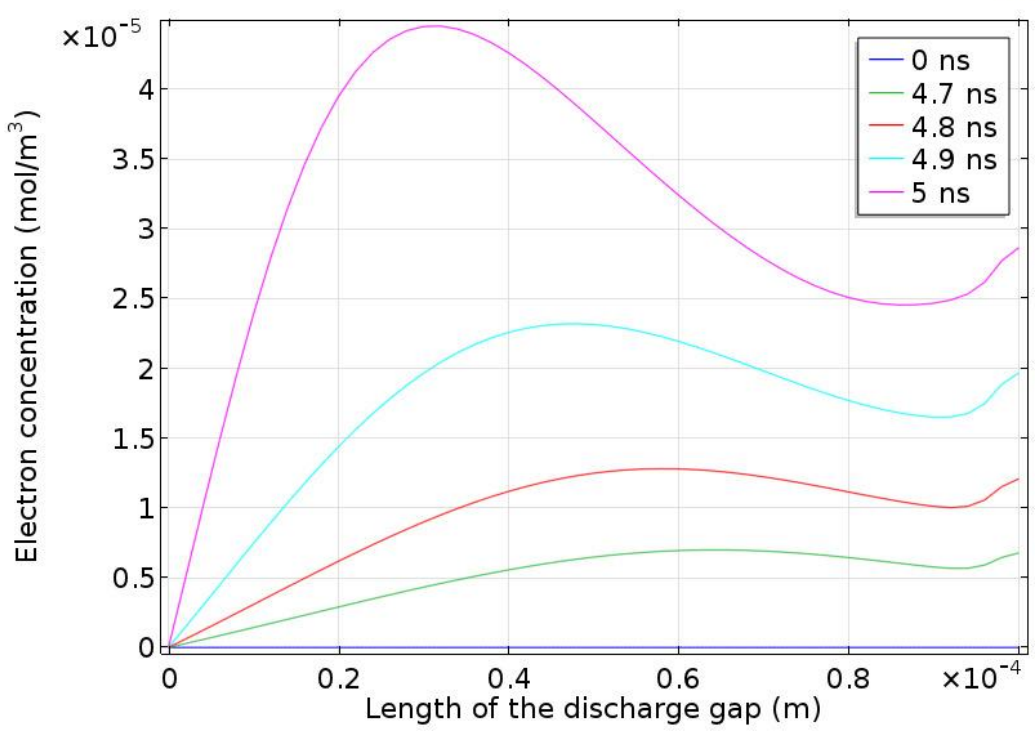

a)

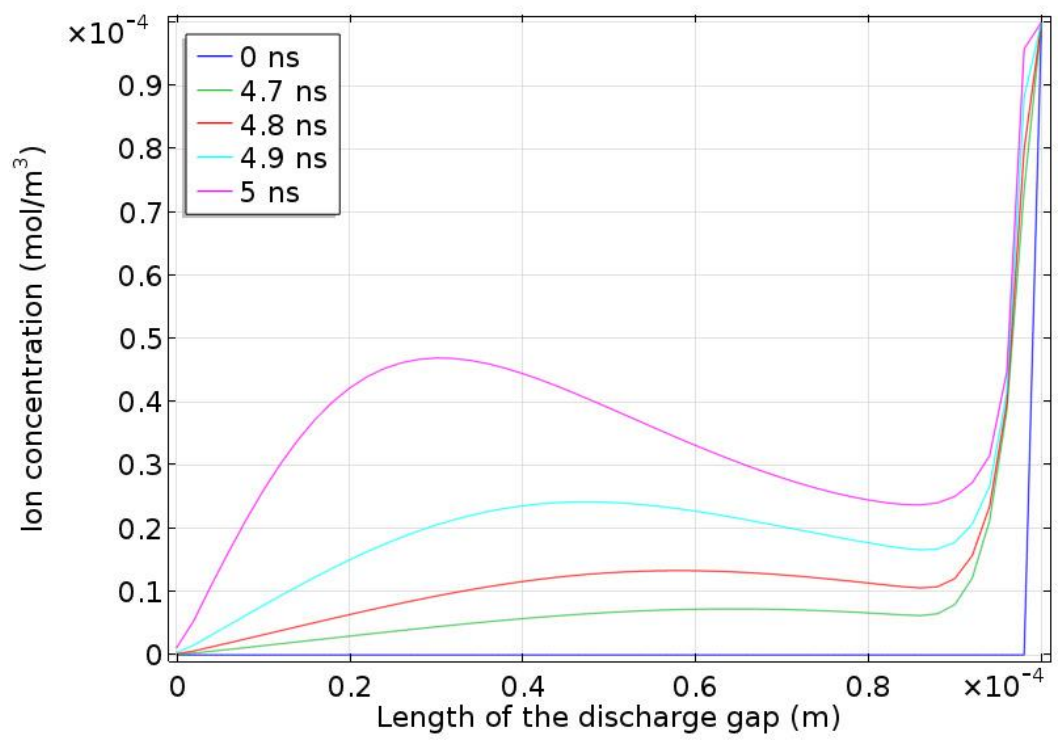

b)

FIGURE 4. 

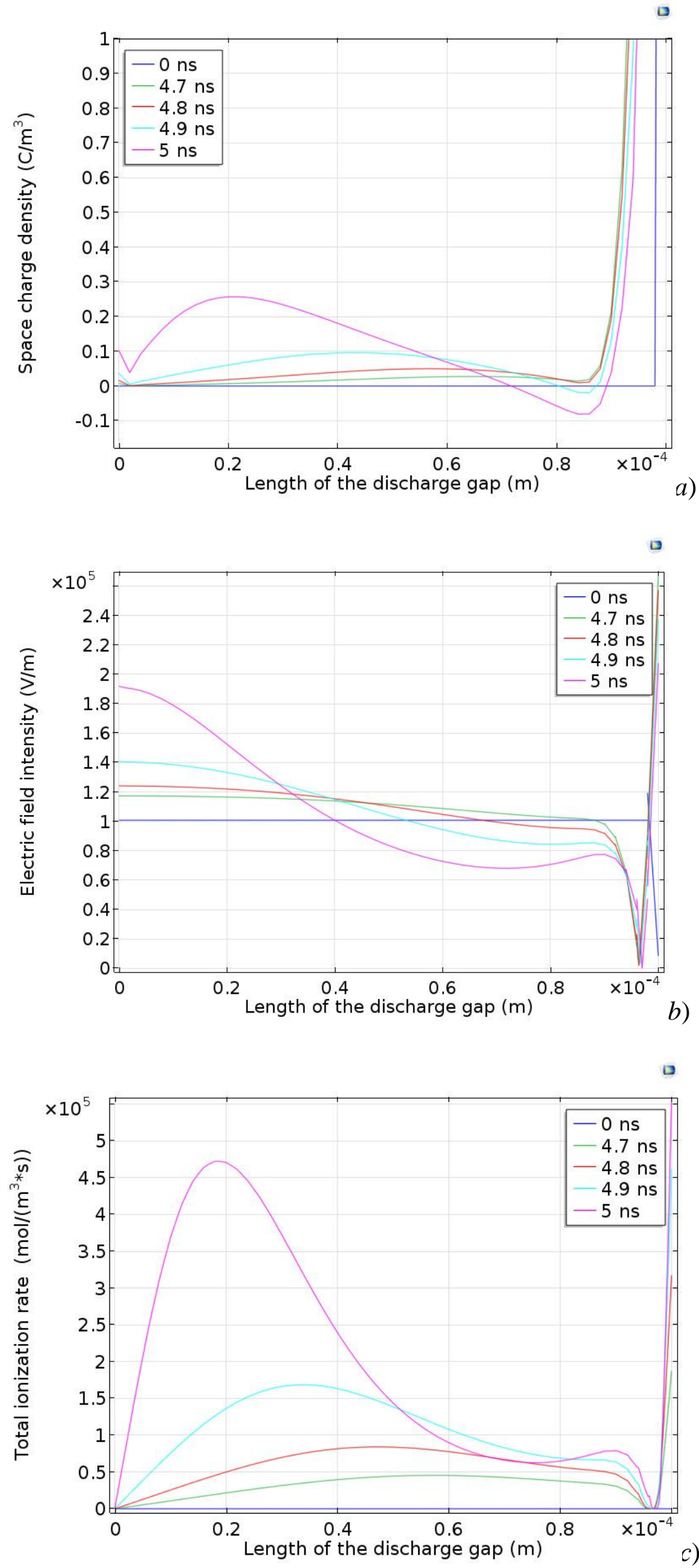

FIGURE 5. 